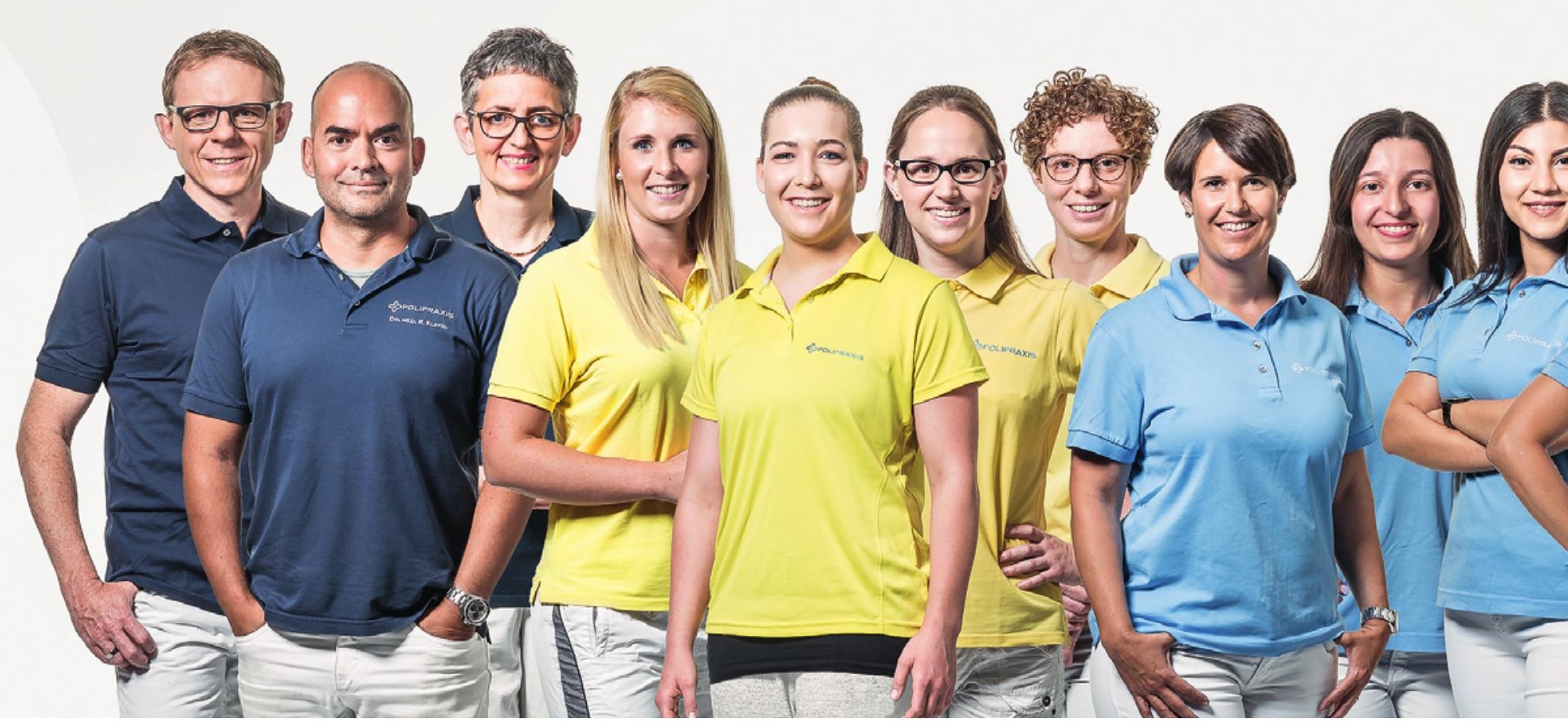

Robert Klingel (zweiter von links) führt zusammen mit zwei Arbeitskollegen drei Gruppenpraxen in der Ostschweiz.

\title{
Ein Bayer in der Schweiz
}

\section{Matthias Scholer}

Online- und Printredaktor SÄZ

Rund ein Drittel der Ärztinnen und Ärzte in der Schweiz kommen aus dem Ausland. Weshalb haben sie sich für ein Leben hierzulande entschieden? Welches sind kulturelle Stolperfallen, die es zu umschiffen gilt? Und was halten sie von der Schweizer Gesundheitspolitik? Diesen und weiteren Fragen möchten wir in der neuen, in loser Folge erscheinenden Artikelserie «Grüezi Schweiz» nachgehen. In dieser Ausgabe stellen wir Robert Klingl vor, der von den kalifornischen Stränden in die beschauliche Ostschweiz wechselte.

\section{Der Weg in die Schweiz}

An San Diegos Strand überkam Robert Klingl das Heimweh. "Mir fehlten die Berge und Seen», erinnert sich der gebürtige Münchner. Klingl sammelte zu dieser Zeit nach seinem Medizinstudium in Berlin praktische Erfahrungen an Kliniken in Südafrika und Kalifornien. Doch die Sehnsucht nach der Heimat veranlasste den jungen Arzt, am kalifornischen Strand sitzend, eine Vielzahl von Blindbewerbungen zu ver- fassen: «Ich sendete diese an verschiedene Kliniken in Bayern, Österreich und in der Schweiz.» Eine davon landete auf der Chirurgie des Spitals Altstätten, Kanton St. Gallen. «Wie es der Zufall wollte, luden mich die Personalverantwortlichen des Spitals für ein Vorstellungsgespräch genau in der Woche ein, in der ich meinen in der Schweiz wohnenden Bruder besuchte», erzählt der Mediziner. Und dann griff ein Rad in das andere: Eigentlich wollte Klingl nur für seine Facharztausbildung im Bereich Allgemeine Innere in der 


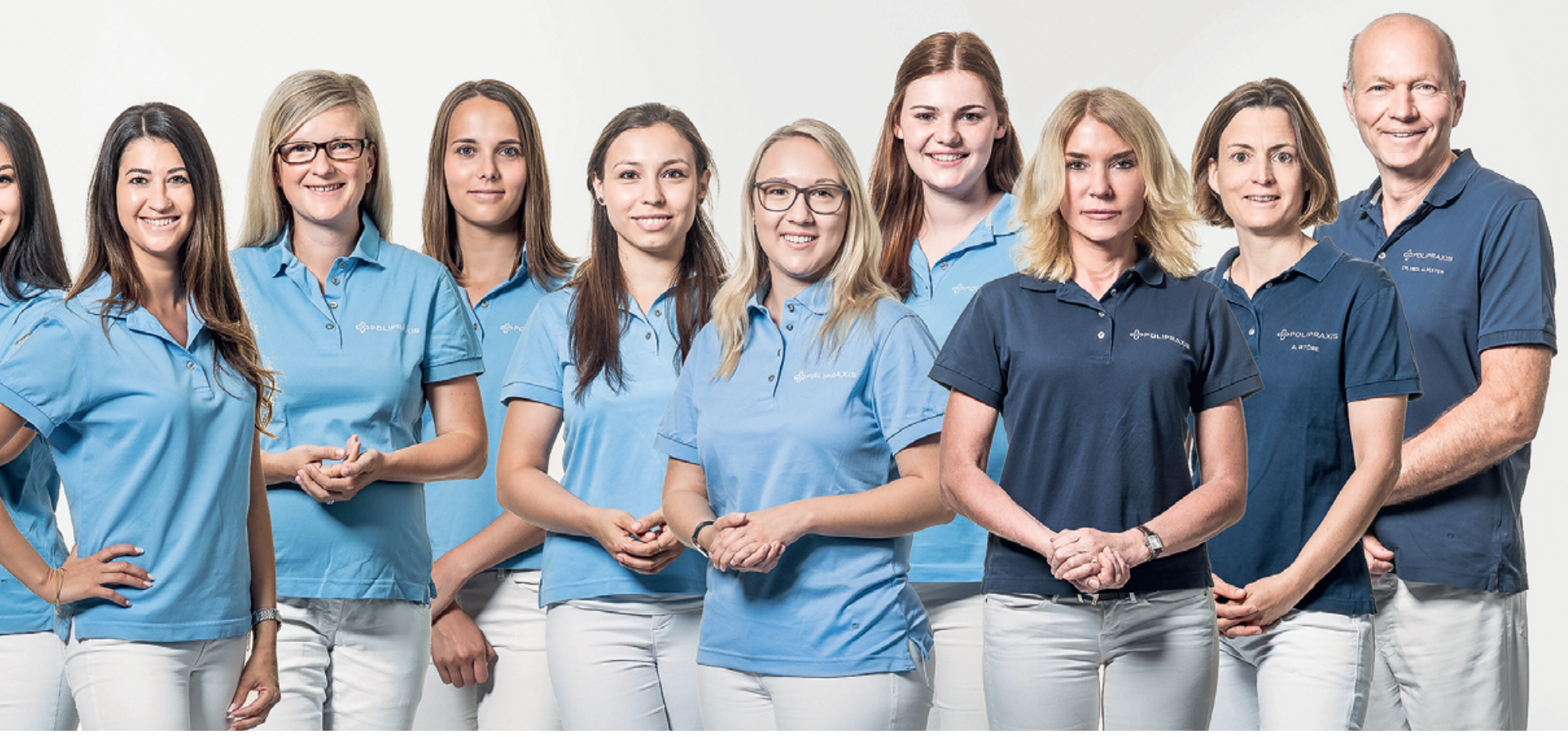

Schweiz bleiben. Doch nach dem Erlangen des Facharztdiploms öffneten sich dem Bayern weitere Türen, um seine berufliche Karriere hierzulande fortzusetzen. "Nach einer Anstellung als Oberarzt am Kantonsspital Baden, übernahm ich 2011 eine leitende Position in einer Praxiskette für Hausarztmedizin", fasst Robert Klingl diesen Teil seines Werdegangs zusammen.

\section{Hoher Ausbildungsstand und vielseitige Arbeit}

Der Hausarztmedizin und der Schweiz blieb Robert Klingl bis heute treu. Und dass er nicht so schnell plant, in seine Heimat zurückzukehren, zeigt sich unter anderem an seinem beruflichen Engagement. Klingl gründete zusammen mit Geschäftspartnern ein Unternehmen, welches an drei Standorten in der Ostschweiz eine Gruppenpraxis betreibt. In diesen steht vor allem die Hausarztmedizin im Mittelpunkt. Doch nicht nur die Grundversorgung der Patienten war bei der Gründung der Praxen ein wichtiger Treiber: «Viele Oberärztinnen und Oberärzte haben genug von 24

Die Tatsache, dass man hierzulande als Arzt in Eigenregie Aspekte in der Grundversorgung mitbeeinflussen kann, ist für Robert Klingl alles andere als selbstverständlich.

Stundendiensten. Sie möchten gerne in die Praxis wechseln und Teilzeit arbeiten. Wir schaffen mit unserem Konzept entsprechende Stellen und leisten gleichzeitig unseren Beitrag an eine gute Grundversorgung in der Region.» Die Tatsache, dass man hierzulande als Arzt in Eigenregie Aspekte in der Grundversorgung

«Schweizer Hausärztinnen und Hausärzte sind auch Teillaborärzte, Teilradiologinnen und Teilapotheker.»

mitbeeinflussen kann, ist für Robert Klingl alles andere als selbstverständlich. Denn in Deutschland wäre dies undenkbar. Und: «In der Schweiz ist der Hausarztberuf vielseitig und anspruchsvoll.» Anders als in seinem Heimatland sitze man hier als Allgemeinmediziner nicht einfach im Anzug und mit dem Kugelschreiber in der Hand am Tisch, um seine Patienten zu triagieren und Rezepte auszustellen. «Schweizer Hausärztinnen und Hausärzte sind auch Teillaborärzte, Teilradiologinnen und Teilapotheker. Wir bieten den Patienten in der Hausarztpraxis eine unglaubliche Fülle an Diagnostik und Therapie an", sagt Klingl begeistert. Er ist überzeugt, dass die Schweizer Hausärztinnen und Hausärzte verglichen mit ihren deutschen Kolleginnen und Kollegen einen sehr hohen Ausbildungsstand haben. Das hänge auch damit zusammen, dass es hierzulande einfacher ist, während der Weiterbildung verschiedene Disziplinen auch in kürzeren Intervallen zu durchlaufen.

Robert Klingl ist denn auch mit der Stossrichtung der Schweizer Gesundheitspolitik grundsätzlich zufrieden. «Ich denke, die TARMED Revision war überfällig. Und die Absicht, High-Tech-Medizin nur in ausgewählten Ballungszentren anzubieten, dafür flächendeckend eine qualitativ hochstehende Grundversorgung anzubieten, ist die richtige Strategie», ist Klingl überzeugt. 


\section{Die Mentalität der Bergvölker}

Und die Schweiz als Einwanderungsland - ein hartes Pflaster? «Ich persönlich hatte nie irgendwelche Schwierigkeiten. Das mag einerseits daran liegen, dass ich seit meiner Jugend Schweizer Freunde habe», sagt Robert Klingl. Lachend fügt er an: «Andererseits bin

\section{Robert Klingl vermutet, dass ein englischer oder französischer Arzt generell freundlicher empfangen würde.}

ich auch kein Deutscher, sondern Bayer». Die Mentalität der "Bergvölker» gleiche sich in vielen Punkten. Zudem reden auch die Bayern einen Dialekt, der nicht immer leicht zu verstehen ist. «Ich fuhr einmal für eine Vertretung direkt von meiner Heimat ins Wallis und vergass, Hochdeutsch zu sprechen. Die erste Patientin schaute mich verdutzt an, weil sie kein Wort verstand. Dies hatte jedoch den Vorteil, dass sie sich dann auch Mühe gab, ihren Walliserdialekt zu entschärfen», erinnert sich Klingl.

Doch auch für den Bayern war es nicht immer einfach: "Als junger Oberassistent hörte ich schon ab und zu seitens des Personals oder der Patienten Bemerkungen wie 'schon wieder ein Deutscher' oder 'der ist doch nur wegen dem Geld hier'. Auch wenn das nur jeder hundertste offen ausspricht, nervt es mit der Zeit und verletzt auch. Schliesslich vertreten diese Leute die
Meinung eines Bevölkerungsteils.» Robert Klingl vermutet, dass ein englischer oder französischer Arzt generell freundlicher empfangen würde. Sein Fazit: «Die Schweiz ist kein Einwanderungsland, in dem man erwarten darf, irgendwann einmal 100 Prozent integriert zu sein. Im besten Fall gelingt dies der zweiten Generation.» Trotzdem fühlt sich Robert Klingl in der Schweiz zu Hause. «Was mich hier hält, ist die flache Hierarchie, die Kollegialität und dass man mit Kompetenzen und nicht mit hierarchischem Gehabe überzeugen muss.» So empfindet der Bayer von seinen Patienten immer wieder das «Du» angeboten - in Deutschland wäre dies ein Affront, in der Schweiz für einen Hausarzt ein Ritterschlag. Auch im Alltag geniesst Klingl, dass man im Vergleich zu Deutschland rücksichtsvoller und anständiger miteinander umgeht - und sei es nur im Strassenverkehr oder beim Bäcker.

Crédit photo:

KONTUR, St. Gallen

En Suisse, un tiers environ des médecins vient de l'étranger Pourquoi ont-ils choisi de vivre ici? Quels sont les pièges culturels qu'ils doivent éviter? Et que pensent-ils de la politique de santé suisse? Nous aimerions répondre à ces questions et à d'autres dans notre nouvelle série d'articles publiés occasionnellement, "Bonjour la Suisse». Pour celle-ci, nous sommes à la recherche de médecins étrangers prêts à nous donner un aperçu de leur vie et de leur quotidien professionnel. Nous nous réjouissons de votre prise de contact à l'adresse mscholer[at]emh.ch. 